\title{
DUALITIES FOR SOME DE MORGAN ALGEBRAS WITH OPERATORS AND LUKASIEWICZ ALGEBRAS
}

\author{
ROBERTO CIGNOLI and MARTA S. DE GALLEGO
}

(Received 3 September 1981, Revised 22 June 1982)

Communicated by J. B. Miller

\begin{abstract}
Algebras $\langle A, \vee, \wedge, \sim, \gamma, 0,1\rangle$ of type $(2,2,1,1,0,0)$ such that $\langle A, \vee, \wedge, \sim, 0,1\rangle$ is a De Morgan algebra and $\gamma$ is a lattice homomorphism from $A$ into its center that satisfies one of the conditions (i) $a \leqslant \gamma a$ or (ii) $a \leqslant \sim a \vee \gamma a$ are considered. The dual categories and the lattice of their subvarieties are determined, and applications to Lukasiewicz algebras are given.
\end{abstract}

1980 Mathematics subject classification (Amer. Math. Soc.): 06 D 30, 03 G 20, 03 G 25, 08 B 15.

\section{Introduction}

Throughout this paper, $\mathbf{A}$ will denote the class of De Morgan algebras and $\mathcal{Q}$ the category of De Morgan algebras and homomorphisms. The class of Kleene algebras (that is De Morgan algebras which satisfy the equation $(a \wedge \sim a) \vee(b$ $\vee \sim b)=b \vee \sim b$ ) will be denoted by $\mathbf{K}$, and the corresponding category by $\mathscr{K}$. For each $A$ in $A, B(A)$ will denote the center of $A$, that is the subalgebra of all complemented elements of $A$, and $K(A)$ the subalgebra of $B(A)$ formed by the elements $a$ such that the De Morgan negation $\sim a$ coincides with the complement of $a$ (see Cignoli and de Gallego (1981)).

Let $A \in \mathbf{A}$. For each $a \in A$, define $K_{a}=\{k \in K(A): a \leqslant k\}$ and $H_{a}=\{k \in$ $K(A): a \leqslant \sim a \vee k\}$. If for each $a$ in $A, K_{a}\left(H_{a}\right)$ has a least element, to be denoted by $\nabla a(\alpha a)$, then $A$ is said to be a $\nabla$-De Morgan ( $\alpha$-De Morgan) algebra. These algebras were introduced in Cignoli and de Gallego (1981) in connection with the theory of $n$-valued Lukasiewicz algebras.

(C) Copyright Australian Mathematical Society 1983 
Let $\mathbf{S}(\mathbf{R})$ be the subclass of the class of $\nabla$-De Morgan ( $\alpha$-De Morgan) algebras, characterized by the property that the map $a \mapsto \nabla a(a \mapsto \alpha a)$ is a lattice homomorphism. Our aim in this paper is to investigate the classes $\mathbf{S}$ and $\mathbf{R}$. After recalling in Section 1 some results on the representation of De Morgan algebras by ordered topological spaces, we show in Section 2 that $\mathbf{S}$ is an equational class that coincides with the class of De Morgan algebras $A$ that are also Stone lattices and are such that the pseudocomplement $a^{*} \in K(A)$ for each $a \in A$. We call the algebras in $\mathbf{S}$ involutive Stone algebras. We characterize the dual spaces of these algebras, and as an application, we prove that the lattice of equational subclasses of $\mathbf{S}$ is a six-element chain, and that the class of three-valued Lukasiewicz algebras can be identified with the class of semi-simple involutive Stone algebras.

In Section 3 we consider the class $\mathbf{R}$, whose elements are called regular $\alpha-D e$ Morgan algebras. We prove that it is an equational class and we develop a duality theory for the algebras in $\mathbf{R}$. We also show that the class of three-valued Lukasiewicz algebras can be identified with the class of semi-simple regular $\alpha$-De Morgan algebras.

Finally, in Section 4 we consider the class $\mathbf{Q}$ of De Morgan algebras endowed with two operators $\alpha$ and $\nabla$ in such a way that $(A, \alpha) \in \mathbf{R}$ and $(A, \nabla) \in \mathbf{S}$. Each of the classes of $n$-valued Lukasiewicz algebras, for $2 \leqslant n \leqslant 5$, is characterized as an equational subclass of $\mathbf{Q}$ and we show that the semi-simple algebras in $\mathbf{Q}$ are exactly the five-valued Lukasiewicz algebras.

We wish to express our gratitude to the referees, whose criticisms to a first draft of this paper strongly influenced its present form.

\section{Priestley's duality for De Morgan algebras}

We assume the reader familiar with the theory of De Morgan and Lukasiewicz algebras, as it is given, for instance, in R. Balbes and P. Dwinger (1974), Chapter XI. The duality theory of De Morgan algebras, based upon the representation of bounded distributive lattices by ordered topological spaces due to Priestley (1970, 1972) (see also Cornish (1975)) was developed by Cornish and Fowler (1977, 1979) (see also Cignoli (1979)).

The following notations will be used systematically throughout this paper.

For each integer $n \geqslant 2, L_{n}$ will denote the $n$-element chain $0<1 /(n-1)<$ $\cdots<(n-2) /(n-1)<1$ with the natural lattice structure and $\sim(j /(n-1))$ $=(n-1-j) /(n-1)$ for $j=0,1, \ldots, n-1$.

Let $X$ be a poset (= partially ordered set) and $Y \subseteq X$. Max $Y(\operatorname{Min} Y)$ will denote the set of maximal (minimal) elements of $Y$ in $X$, and $[Y)((Y])$ the set of all $x$ in $X$ such that $y \leqslant x(x \leqslant y)$ for some $y \in Y$. Y is increasing (decreasing) if $Y=[Y)(Y=(Y])$. 
Recall that a totally order-disconnected topological space is a triple $(X, \leqslant, \tau)$ such that $(X, \leqslant)$ is a poset, $(X, \tau)$ is a topological space and given $x, y$ in $X$, with $x \neq y$, there is a clopen (= closed and open) increasing set $U$ such that $x \in U$ and $y \notin U$. The category of compact totally order-disconnected spaces and order-preserving continuous functions will be denoted by $\mathcal{T}$. As usual, we are going to denote the objects in $\mathscr{T}$ by its underlying sets $X$. For each object $X$ in $\mathscr{T}$, $\mathrm{D}(X)$ will denote the set of increasing clopen subsets of $X$.

We shall denote by $\mathbf{G}$ the class of all pairs $(X, g)$ such that $X$ is an object in $\mathcal{T}$ and $g: X \rightarrow X$ is an involutorial homeomorphism that is also an order-isomorphism from $X$ onto its order-dual. If $(X, g)$ and $\left(X^{\prime}, g^{\prime}\right)$ are in $\mathbf{G}, f: X \rightarrow X^{\prime}$ is said to be a G-function if $f$ is a morphism in $\sigma$ and $f g=g^{\prime} f$. The category whose objects are the elements of $\mathbf{G}$ and whose morphisms are the $\mathbf{G}$-functions will be denoted by $\mathcal{G}$.

If $(X, g)$ is in $\mathbf{G}$ and $U \in \mathbf{D}(X)$, define $\sim U=X \backslash g(U)$. Then $\langle\mathbf{D}(X), \cup, \cap, \sim, \varnothing, X\rangle$ is a De Morgan algebra, which we are going to denote by $\mathscr{D}(X, g)$. If $f$ is a G-function from $(X, g)$ into $\left(X^{\prime}, g^{\prime}\right)$, define $\mathscr{D}(f)$ : $\mathbf{D}\left(X^{\prime}\right) \rightarrow \mathbf{D}(X)$ by the prescription $\mathscr{D}(f)\left(U^{\prime}\right)=f^{-1}\left(U^{\prime}\right)$. It follows that $\mathscr{Q}(f)$ is a De Morgan homomorphism from $\mathscr{D}\left(X^{\prime}, g^{\prime}\right)$ into $\mathscr{D}(X, g)$. Thus $\mathscr{D}$ is a functor from $\mathcal{G}$ into the opposite category of $\mathcal{Q}$. Note that $K(\mathscr{D}(X, g))=\{W \in$ $\mathrm{D}(X): g(W)=W\}$.

If $A$ is a De Morgan algebra, let $\operatorname{Pr}(A)$ denote the pair $(\mathbf{X}(A), g)$, where $\mathbf{X}(A)$ is the set of prime filters of $A$, ordered by inclusion and with the topology having as a subbasis the sets of the form $\sigma(a)=\{P \in \mathbf{X}(A): a \in P\}$ and $\mathbf{X}(A) \backslash \sigma(a)$ for each $a$ in $A$, and $g: \mathbf{X}(A) \rightarrow \mathbf{X}(A)$ is defined by the prescription: $g(P)=$ $A \backslash\{\sim a: a \in P\}$. It follows that $(\mathbf{X}(A), g)$ is in G. If $h: A \rightarrow A^{\prime}$ is a morphism in $Q$, define $\operatorname{Pr}(h): \mathbf{X}\left(A^{\prime}\right) \rightarrow \mathbf{X}(A)$ as $\mathscr{P} r(h)\left(P^{\prime}\right)=h^{-1}\left(P^{\prime}\right)$. Then $\mathscr{P} r(h)$ is a Gfunction and $\mathscr{P} r$ defines a functor from the category $\mathscr{Q}$ into the opposite category of $\mathcal{G}$.

Moreover, if $A$ is a De Morgan algebra, the map $\sigma: A \rightarrow \mathscr{D}(\mathcal{P} r(A))$ is a De Morgan algebra isomorphism, and if $(X, g) \in \mathbf{G}$, the function $\varepsilon: X \rightarrow$ $\operatorname{Pr}(\mathscr{D}(X, g))$ defined by the prescription: $\varepsilon(x)=\left\{U^{\prime} \in \mathbf{D}(X): x \in U\right\}$ is an order-preserving homeomorphism. Thus the functors $\mathcal{P} r$ and $\mathscr{D}$ establish a duality between the categories $\mathscr{Q}$ and $\mathcal{G}$.

If $(X, g) \in \mathbf{G}$, we set $X_{1}=\{x \in X: x \leqslant g(x)\}$ and $X_{2}=g\left(X_{1}\right)=\{x \in X$ : $g(x) \leqslant x\}$.

It is well known that a De Morgan algebra $A$ belongs to $\mathbf{K}$ if and only if for each prime filter $P$ of $A, P \subseteq g(P)$ or $g(P) \subseteq P$. Thus if we denote by $\mathbf{G}_{K}$ the class of pairs $(X, g)$ in $\mathbf{G}$ such that $X=X_{1} \cup X_{2}$, and by $\mathcal{G}_{K}$ the full subcategory of $\mathcal{G}$ whose objects are the elements of $\mathbf{G}_{K}$, then it follows at once that the restrictions of the functors $\mathcal{P r}$ and $\mathscr{D}$ to the subcategories $\mathcal{K}$ of $\mathcal{Q}$ and $\mathcal{G}_{K}$ of $\mathcal{G}$ respectively, establish a duality between $\mathscr{K}$ and $\mathcal{G}_{K}$. 
Let $(X, g) \in \mathbf{G}$. If $Y \subseteq X$, we shall denote by $\Theta(Y)$ the relation defined on $\mathbf{D}(X)$ as follows: $(U, V) \ddot{\in} \Theta(Y)$ if and only if $U \cap Y=V \cap Y$. We shall say that $Y \subseteq X$ is an involutive set if $g(Y)=Y$.

The following results are essentially due to Cornish and Fowler (1979), and are based on results of Priestley $(1972,1975)$ on distributive lattices:

1.1. Lemma. (i) If $(X, g) \in \mathbf{G}$ and $Y$ is a closed involutive subset of $X$, then $\Theta(Y)$ is a De Morgan algebra congruence on $\mathscr{D}(X, g)$. Moreover, if $\pi: \mathcal{D}(X, g) \rightarrow$ $\mathscr{D}(X, g) / \Theta(Y)$ is the natural projection, then

$$
Y=\varepsilon^{-1}(\mathscr{P} r(\pi)(\mathscr{P} r(\mathscr{D}(X, g) / \Theta(Y)))) .
$$

(ii) Let $A \in \mathbf{A}$ and $\Theta$ be a congruence on $A$. If $\pi: A \rightarrow A / \Theta$ is the natural projection, then $Y=\operatorname{PP}(\pi)(\mathscr{P} r(A))$ is a closed involutive subset of $\operatorname{Pr}(A)$, and $(a, b) \in \Theta$ if and only if $(\sigma(a), \sigma(b)) \in \Theta(Y)$.

Recall that an equational class $\mathbf{C}$ of algebras is said to be congruence-distributive if the lattice of congruences of each algebra in $\mathbf{C}$ is distributive. For an equational class $\mathrm{C}$, let $\mathrm{Si}(\mathrm{C})$ consist of precisely one algebra from each of the isomorphism classes of the subdirectly irreducible algebras in $\mathbf{C}$. By combining results of Jónsson (1967) on congruence-distributive classes with the well known Birkhoff's theorem on the representation of finite distributive lattices by sets, Davey (1979) obtained the following result, which we quote for further reference:

1.2. THEOREM. Let $\mathbf{C}$ be a congruence-distributive equational class generated by a finite set of finite algebras, and order $\mathrm{Si}(\mathrm{C})$ by $A \leqslant B$ if and only if $A$ is $a$ homomorphic image of a subalgebra of $B$. Then the lattice of equational subclasses of $\mathrm{C}$ is a finite distributive lattice isomorphic to the lattice of decreasing subsets of the poset $\mathbf{S i}(\mathbf{C})$.

\section{Involutive Stone algebras}

Recall that $\mathbf{S}$ denotes the class of $\nabla$-De Morgan algebras $A$ such that the map $a \rightarrow \nabla a$ is a lattice homomorphism from $A$ into $K(A)$.

2.1. Theorem. $\mathbf{S}$ is an equational class. Indeed, a De Morgan algebra $A \in \mathbf{S}$ if and only if there is an operator $\nabla: A \rightarrow A$ satisfying the following equations:

(S1) $\nabla 0=0$

(S2) $a \wedge \nabla a=a$

(S3) $\nabla(a \wedge b)=\nabla a \wedge \nabla b$

(S4) $\sim \nabla a \wedge \nabla a=0$. 
Proof. It is plain that the operator $\nabla$ satisfies (S1)-(S4) for each $A \in \mathbf{S}$. Conversely, suppose that $A \in \mathbf{A}$ and $\nabla: A \rightarrow A$ fulfills conditions (S1)-(S4). Let $k \in K(A)$. (S1) and (S3) imply that $0=\nabla(k \wedge \sim k)=\nabla k \wedge \nabla \sim k$, and since, by (S4), $\nabla a \in K(A)$ for each $a \in A$, we have that $\nabla k \leqslant \sim \nabla \sim k$. But it follows from (S2) that $k \leqslant \nabla k$ and $\sim \nabla \sim k \leqslant \sim \sim k=k$. Consequently, $\nabla k=k$. In particular, $\nabla \nabla a=\nabla a$ for each $a \in A$, and then $\nabla$ is a closure operator defined on $A$ such that $\nabla(A)=K(A)$. It is well known that this implies that $\nabla a$ is the least element in $K_{a}$ and moreover, since $K(A)$ is a sublattice of $A$, that $\nabla(a \vee b)$ $=\nabla a \vee \nabla b$ for each $a, b$ in $A$. Therefore, $A \in \mathbf{S}$.

If $A \in \mathrm{S}$, then $\sim \nabla a=a^{*}$ is the pseudocomplement of $A$ (see J. Varlet (1968)), and $a^{* *}=\sim \nabla \sim \nabla a=\nabla a$. Since $a^{*} \vee a^{* *}=\sim \nabla a \vee \nabla a=1, A$ is a Stone lattice. The dual pseudocomplement $a^{+}$of $a$ also exists, in fact, $a^{+}=\nabla \sim a$, and $A$ is also a dual Stone lattice. Therefore, $\mathbf{S}$ is a subclass of the class of double Stone lattices. More precisely, it is a proper subclass: for instance, $\mathbf{2} \oplus \mathbf{B}_{2} \oplus \mathbf{1}$, where $\mathbf{B}_{n}$ is the Boolean algebra with $n$ atoms, is a double Stone lattice that does not admit a De Morgan algebra structure.

2.2. Remark. It is well known that in a Stone lattice $A, a \in B(A)$ if and only if $a=a^{* *}$. Consequently, if $A$ is a De Morgan algebra that is also a Stone lattice, and if we define $\nabla a=a^{* *}$, we have that the following conditions are equivalent: (i) The algebra $\langle A, \vee, \wedge, \sim, \nabla, 0,1\rangle \in \mathbf{S}$, (ii) $a^{*}=\sim\left(a^{* *}\right)$ for each $a \in A$, and (iii) $B(A)=K(A)$.

Motivated by the above remarks, we shall call the algebras in $\mathbf{S}$ involutive Stone algebras. The category of involutive Stone algebras and homomorphisms will be denoted by $\mathcal{S}$.

The algebras $L_{n}$ are important examples of involutive Stone algebras. Note that for each $n \geqslant 2 \nabla(j /(n-1))=1$ for $1 \leqslant j \leqslant n-1$ and $=0$ for $j=0$. If $\left\langle A, \vee, \wedge, \sim, \sigma_{1}^{n}, \ldots, \sigma_{n-1}^{n}, 0,1\right\rangle$ is an $n$-valued Lukasiewicz algebra, then the reduct $\left\langle A, \vee, \wedge, \sim, \sigma_{n-1}^{n}, 0,1\right\rangle \in \mathbf{S}$ (Cignoli and de Gallego (1981)). In particular, when $n=3$ we have that $\left\langle A, \vee, \wedge, \sim, \sigma_{2}^{3}, 0,1\right\rangle \in \mathbf{S}$ and that the operator $\sigma_{2}^{3}$ satisfies the relation $a \wedge \sigma_{2}^{3} \sim a \leqslant b \vee \sim \sigma_{2}^{3} b$ for each $a, b$ in $A$. On the other hand, if $A \in \mathbf{S}$ and the relation $a \wedge \nabla \sim a \leqslant b \vee \sim \nabla b$ holds in $A$, then it is easy to check that the system $\langle A, \vee, \wedge, \sim, \triangle, \nabla, 0,1\rangle$ is a three-valued Lukasiewicz algebra, where $\Delta=\sim \nabla \sim$. Therefore we have:

2.3. Proposition. The class of three-valued Lukasiewicz algebras coincides with the class of involutive Stone algebras that satisfy the equation (ES3) $(a \wedge \nabla \sim a) \vee$ $(b \vee \sim \nabla b)=b \vee \sim \nabla b$. 
We shall denote by $\mathbf{G}_{S}$ the class of pairs $(X, g) \in \mathbf{G}$ satisfying the following condition: (S) For each $U \in \mathbf{D}(X),(U]$ is open and $g\left((U)=(U]\right.$. If $X, X^{\prime}$ are in $\mathbf{G}_{S}$, a G-function $f: X \rightarrow X^{\prime}$ is called an S-function if $f(\operatorname{Max} X) \subseteq \operatorname{Max} X^{\prime}$. The category whose objects are the pairs in $\mathbf{G}_{S}$ and whose morphisms are the S-functions will be denoted by $\mathcal{G}_{s}$.

Let $A \in \mathbf{S}$ and $(X, g)=\operatorname{Pr}(A)$. Since $A$ is a Stone lattice, it follows from Priestley (1974, Proposition 2), that $\sigma(\nabla a)=\sigma\left(a^{* *}\right)=(\sigma(a)]$ for each $a \in A$, and since $\nabla a \in K(A)$, we have that $g((\sigma(a)])=(\sigma(a)]$. Therefore, $(X, g) \in \mathbf{G}_{s}$. Conversely, let $(X, g) \in \mathbf{G}_{S}$. Condition (S) implies that $(U]$ is open and increasing for each $U \in \mathbf{D}(X)$, and then it follows again from Priestley (1974, Proposition 2), that $\mathbf{D}(X)$ is a Stone lattice and that $(U]=U^{* *}=\nabla U$. Hence, by taking into account Remark 2.2, we obtain that $\mathscr{D}_{S}(X, g)=\langle\mathbf{D}(X), \cup, \cap$, $\sim, \nabla, X, \varnothing\rangle \in \mathbf{S}$. Moreover, since it follows from Remark 2.2 that the morphisms in $\subseteq$ are the De Morgan algebra homomorphisms that preserve the pseudocomplement, the characterization of the dual maps of Stone algebra homomorphisms given in Priestley (1974, Proposition 5), implies that the dual maps of the morphisms in $\delta$ are exactly the $\mathbf{S}$-functions.

It is now easy to complete the proof of the following theorem, where $\mathcal{P r}_{\boldsymbol{s}}$ denotes the restriction of the functor $\mathscr{P r}$ to the subcategory $\mathcal{S}$ of $\mathcal{Q}$, and $\mathscr{D}_{S}(f)=\mathscr{Q}(f)$ for each S-function $f$ :

2.4. THEOREM. The categories $\mathcal{S}_{S}$ and $\varsigma^{\mathrm{op}}$ are naturally equivalent. More precisely, the composite functors $\mathscr{D}_{S} \mathcal{P} r_{S}$ and $\mathscr{P} r_{S} \mathscr{D}_{S}$ are naturally equivalent to the identity functors of $\mathcal{S}$ and $\mathcal{G}_{S}$ respectively. The corresponding unit is $\varepsilon$ and the co-unit $\sigma$.

Let $(X, g) \in \mathbf{G}_{\boldsymbol{S}}$. An involutive closed subset $Y$ of $X$ is called an $\mathbf{S}$-set if $[Y) \cap \operatorname{Max} X \subseteq Y$.

It was proved in Priestley (1975, Section 4) (see also Davey (1978)) that if $X$ is the dual space of a pseudocomplemented distributive lattice, then the lattice congruences that preserve the pseudocomplement are of the form $\Theta(Y)$, where $Y$ is a closed subset of $X$ such that $[Y] \cap \operatorname{Max} X \subseteq Y$. From this result, Lemma 1.1 and Remark 2.2 we have at once:

2.5. TheOREM. Let $A \in \mathbf{S}$ and $(X, g)=\mathcal{P} r_{S}(A)$. The map $Y \mapsto \Theta(Y)$ establishes an isomorphism from the lattice of $\mathbf{S}$-sets of $X$ onto the order dual of the lattice of congruences of $A$.

Let $(X, g) \in \mathbf{G}_{s}$. Since $X$ is the dual space of a Stone lattice, it is well known (see, for instance, Priestley (1974, Proposition 3)) that for each $x \in X$ there is exactly one $n_{x} \in \operatorname{Max} X$ such that $x \leqslant n_{x}$. Note that $n_{g(x)}=n_{x}$ for each $x \in X$. 
For, if $g(x) \$ n_{x}$, then we would find an $U \in \mathbf{D}(X)$ such that $g(x) \in U$ and $n_{x} \notin U$, and since $n_{x} \in \operatorname{Max} X$, we would have that $n_{x} \notin(U]$. But this is not possible, because by condition (S) $g((U])=(U]$, and then $(U]$ has to contain all the elements comparable with $x$. Consequently, if for each $x \in X$ we define $Y_{x}=\left\{x, n_{x}, g(x), g\left(n_{x}\right)\right\}$, then $Y_{x}$ is an S-set. Write $g_{x}$ for the restriction of $g$ to $Y_{x}$. Since $Y_{x}=\varepsilon^{-1}\left(\mathscr{P}_{S} r_{S}(\pi)\left(\mathscr{Q}_{S}(X, g) / \Theta\left(Y_{x}\right)\right)\right)$, it follows that $\left(Y_{x}, g_{x}\right) \in \mathscr{G}_{S}$ and that $\mathscr{Q}_{S}\left(Y_{x}, g_{x}\right) \cong \mathscr{Q}_{S}(X, g) / \Theta\left(Y_{x}\right)$. Moreover, since $\cup_{x \in X} Y_{x}=X$, the above theorem implies that:

2.6. LemMa. If $A \in \mathbf{S}$ and $(X, g)=\operatorname{Pr}(A)$, then $A$ is a subdirect product of the family $\left\{\mathscr{D}_{S}\left(Y_{x}, g_{x}\right)\right\}_{x \in X}$.

For each $x \in X$ we have the following possible cases:

(1) $g\left(n_{x}\right)=g(x)=x=n_{x}$

(2a) $g\left(n_{x}\right)=g(x)<x=n_{x}$

(2b) $g\left(n_{x}\right)=x<g(x)=n_{x}$

(3) $g\left(n_{x}\right)<g(x)=x<n_{x}$

(4a) $g\left(n_{x}\right)<g(x)<x<n_{x}$

(4b) $g\left(n_{x}\right)<x<g(x)<n_{x}$

(5) $\quad g\left(n_{x}\right)<x, g(x)<n_{x}$ and $x$ is not comparable with $g(x)$.

Note that in case (i), $1 \leqslant i \leqslant 4, \mathscr{D}_{S}\left(Y_{x}, g_{x}\right) \cong L_{i+1}$. In case (5), $\mathscr{D}_{S}\left(Y_{x}, g_{x}\right) \cong S_{6}$, where $S_{6}$ is the six-element involutive Stone algebra obtained by adding a new zero and a new unit to the Boolean algebra with two atoms, $a, b$, and with determined by the prescriptions $\sim a=a$ and $\sim b=b$.

Therefore we have:

2.7. Lemma. If $\left|Y_{x}\right|=j, j=1,2,3$, then $\mathscr{D}_{S}\left(Y_{x}, g_{x}\right) \cong L_{j+1}$. If $\left|Y_{x}\right|=4$, and $x$ is comparable with $g(x)$, then $\mathscr{Q}_{S}\left(Y_{x}, g_{x}\right) \cong L_{5}$. If $x$ and $g(x)$ are not comparable, then $\mathscr{D}_{S}\left(Y_{x}, g_{x}\right) \cong S_{6}$.

2.8. THEOREM. The subdirectly irreducible algebras in the equational class $\mathbf{S}$ are $L_{i}$, for $2 \leqslant i \leqslant 5$, and $S_{6}$. The simple algebras are $L_{2}$ and $L_{3}$.

Proof. It is easy to check that the algebras $L_{2}$ and $L_{3}$ are simple and that each of $L_{4}, L_{5}$ and $S_{6}$ has exactly one non-trivial congruence, given by the $\mathbf{S}$-set $Y=\operatorname{Min} X \cup \operatorname{Max} X=g(\operatorname{Max} X) \cup \operatorname{Max} X$, where $(X, g)$ denotes the corresponding Priestley space. Consequently, the algebras $L_{i}, 2 \leqslant i \leqslant 5$ and $S_{6}$ are subdirectly irreducible. Suppose now that $A$ is subdirectly irreducible in $\mathbf{S}$. It follows from the above two lemmas that $A$ is a subdirect product of the algebras $L_{i}(2 \leqslant i \leqslant 5)$ and $S_{6}$. Since $A$ is subdirectly irreducible, we must have that either $A \cong L_{i}$ for some $2 \leqslant i \leqslant 5$, or $A \cong S_{6}$. 
It follows at once from Theorems 2.5 and 2.8 that $\mathbf{S}$ is the congruence-distributive equational class generated by the algebras $L_{i}, 2 \leqslant i \leqslant 5$ and $S_{6}$. Moreover, since all the $L_{i}(2 \leqslant i \leqslant 5)$ are subalgebras of $S_{6}$, and $L_{2}$ is a subalgebra of each of the other $L_{i}$, and $L_{3}, L_{4}$ are subalgebras of $L_{5}$, and $L_{3}$ is a homomorphic image of $L_{4}$ (under the homomorphism $h$ determined by the prescription $h(1 / 3)=h(2 / 3)$ $=1 / 2$ ), it follows from Theorem 1.1 that the lattice of equational subclasses of $\mathbf{S}$ is the chain $\mathbf{S}_{1} \subset \mathbf{S}_{2} \subset \cdots \subset \mathbf{S}_{6}=\mathbf{S}$, where $\mathbf{S}_{1}$ is the class of one-element algebras, $\mathbf{S}_{6}$ is the equational subclass of $\mathbf{S}$ generated by $S_{6}$ and $\mathbf{S}_{i}$ is the equational subclass of $\mathbf{S}$ generated by $L_{i}$, for $2 \leqslant i \leqslant 5$.

It is worthwhile to point out that the lattice of equational subclasses of the class of double Stone algebras is a four-element chain (Katriňák (1974), Corollary 2), and that the lattice of equational subclasses of the class of Stone algebras is a three-element chain (Balbes and Dwinger (1974), Chapter VIII, section 7, Theorem 1).

Recall that an algebra is said to be semi-simple if it is a subdirect product of simple algebras. Note that $\mathbf{S}_{3}$ is the class of semi-simple algebras in $\mathbf{S}$.

2.9. ThEOREM. The class of three-valued Lukasiewicz algebras coincides with $\mathbf{S}_{3}$. Thus, the class of three-valued Lukasiewicz algebras can be characterized as the class of semi-simple involutive Stone algebras.

Proof. According to Proposition 2.3, the class of three-valued Lukasiewicz algebras can be identified with the equational subclass $\mathbf{H}$ of $\mathbf{S}$ characterized by the equation (ES3). Since $L_{3} \in \mathbf{H}$, we have that $\mathbf{S}_{3} \subseteq \mathbf{H}$. On the other hand, since the only algebras $L_{i}$ that admit a three-valued Lukasiewicz algebra structure are $L_{2}$ and $L_{3}$, it follows that the subdirectly irreducible algebras in $\mathbf{S}$ that belong to $\mathbf{H}$ are $L_{2}$ and $L_{3}$. Therefore $\mathbf{H} \subseteq \mathbf{S}_{3}$.

\section{Regular $\alpha$-De Morgan algebras}

A De Morgan algebra $A$ is said to be an $\alpha$-De Morgan algebra if for each $a \in A$, the set $H_{a}=\{k \in K(A): a \leqslant \sim a \vee k\}$ has a least element, to be denoted by $\alpha a$. If $A$ is an $\alpha$-De Morgan algebra, we define $\beta a=\sim \alpha \sim a$ for each $a \in A$.

3.1. LeMma. The following properties hold for each prime filter $P$ of an $\alpha$-De Morgan algebra $A$ :

(i) $P \subseteq g(P)$ if and only if $a \in P$ implies $\alpha a \in P$

(ii) $g(P) \subseteq P$ if and only if $\beta a \in P$ implies $a \in P$. 
Proor. (i) Suppose $P \subseteq g(P)$ and let $a \in P$. Since $\sim a \notin P$ and $\alpha a \vee \sim \alpha a=1$, we have that $\alpha a \in P$. To prove the converse, note first that since $\alpha a=0$ if and only if $a \leqslant \sim a$, we have that $\alpha(a \wedge \sim a)=0$ for each $a \in A$. Suppose now that $P \subseteq \alpha^{-1}(P)$ and let $a \in P$. If $a \notin g(P)$, we would have that $a \wedge \sim a \in P$, and consequently, $0=\alpha(a \wedge \sim a) \in P$. But this is not possible, because, by definition, prime filters are proper. The proof of (ii) is analogous.

3.2. Proposition. The following are equivalent conditions in any $\alpha-D e$ Morgan algebra $A$, where $a, b$ denote variables in $A$ :

(i) $\alpha(a \vee b)=\alpha a \vee \alpha b$

(ii) $a \wedge \sim \alpha a \leqslant b \vee \alpha \sim b$

(iii) $A$ is a Kleene algebra.

Proof. (i) $\Rightarrow$ (ii): Suppose that (ii) does not hold in $A$. Then there are elements $a$ and $b$ in $A$ such that $a \wedge \sim \alpha a \neq b \vee \alpha \sim b$. By the well known Birkhoff-Stone theorem, we can find a prime filter $P$ of $A$ such that $a \wedge \sim \alpha a \in P$ and $b \vee \alpha \sim b \notin P$. These conditions imply that:

$$
\text { (1) } \alpha a \vee \alpha \sim b \notin P, \quad \text { (2) } a \vee \sim b \in P \text {, and }
$$

(3) $\sim a \wedge b \notin P$.

Since $(\sim a \wedge b) \vee \alpha(a \vee \sim b)=\sim(a \vee \sim b) \vee \alpha(a \vee \sim b) \geqslant a \vee \sim b$, it follows from (2) and (3) that $\alpha(a \vee \sim b) \in P$, and taking into account (1), it follows that $\alpha(a \vee \sim b) \$ \alpha a \vee \alpha \sim b$. Thus (i) does not hold in $A$.

(ii) $\Rightarrow$ (iii): We have to prove that condition (ii) implies that for each prime filter $P$ of $A, P \subseteq g(P)$ or $g(P) \subseteq P$. Suppose $P \nsubseteq g(P)$. Then, by Lemma 3.1(i) we can find an $a \in A$ such that $a \in P$ and $\alpha a \notin P$. Since $\alpha a \vee \sim \alpha a=1$, we have that $\sim \alpha a \in P$. Thus, $a \wedge \sim \alpha a \in P$ and (ii) yields that $b \vee \sim \beta b \in P$ for each $b \in A$. Hence, by taking into account that $\beta b \wedge \sim \beta b=0$, we obtain from Lemma 3.1(ii) that $g(P) \subseteq P$.

(iii) $\Rightarrow$ (i): This was proved in Cignoli and de Gallego (1981, Proposition 2.1 (vii)).

We call an $\alpha$-De Morgan algebra $A$ regular if $\alpha: A \rightarrow A$ is a lattice homomorphism. The class of regular $\alpha$-De Morgan algebras will be denoted by $R$ and the corresponding category by $R$.

3.3. THEOREM. The class $\mathbf{R}$ of regular $\alpha-D e$ Morgan algebras is equational. More precisely, a De Morgan algebra $A \in \mathbf{R}$ if and only if there is an operator $\alpha: A \rightarrow A$ satisfying the following equations:

$$
\begin{aligned}
& \text { (R1) } \sim \alpha a \vee \alpha a=1 \\
& \text { (R2) }(\sim a \vee \alpha a) \wedge a=a
\end{aligned}
$$


(R3) $\alpha a \wedge \alpha \sim a=0$

(R4) $\alpha(a \vee b)=\alpha a \vee \alpha b$

(R5) $\alpha(a \wedge b)=\alpha a \wedge \alpha b$.

Proof. Note first that since $\alpha(a \wedge \sim a)=0$ (see the proof of Lemma 3.1) we have that if $\alpha$ satisfies (R5) then it also satisfies (R3). Consequently, equations (R1)-(R5) hold in each $A \in \mathbf{R}$. Conversely, let $A \in \mathbf{A}$ and suppose that $\alpha: A \rightarrow A$ satisfies (R1)-(R5). Conditions (R1) and (R2) imply that $\alpha a \in H_{a}$ for each $a$ in $A$. Moreover, we have that $\alpha k=k$ for each $k \in K(A)$. Indeed, since $k \wedge \sim k=0$, it follows from (R2) that $\alpha k=\alpha k \vee(k \wedge \sim k)=(\alpha k \vee k) \wedge(\alpha k \vee \sim k) \geqslant$ $(\alpha k \vee k) \wedge k=k$. The same argument applied to $\sim k$ yields $\alpha \sim k \geqslant \sim k$, and taking into account (R3), we obtain that $k \leqslant \alpha k \leqslant \sim \alpha \sim k \leqslant \sim \sim k=k$. Suppose now that $k \in K(A)$ and $a \leqslant \sim a \vee k$. From (R4) and the equality $\alpha k=k$ it follows that $\alpha a \leqslant \alpha \sim a \vee k$, and (R3) implies that $\alpha a \leqslant k$. Therefore $\alpha a$ is the least element in $H_{a}$.

Important examples of regular $\alpha$-De Morgan algebras are the chains $L_{n}$. Note that for each $n \geqslant 2, \alpha(j /(n-1))=0$ if $2 j \leqslant n-1$ and $=1$ otherwise. If $A$ is an $n$-valued Lukasiewicz algebra, then the reduct $\left\langle A, \vee, \wedge, \sim, \sigma_{t}^{n}, 0,1\right\rangle \in \mathbf{R}$, where $t=(n-1) / 2$ if $n$ is odd and $t=n / 2$ if $n$ is even (Cignoli and de Gallego (1981)). In particular, when $n=3$, we have that $\left\langle A, \vee, \wedge, \sim, \sigma_{1}^{3}, 0,1\right\rangle \in \mathbf{R}$, and the operation $\sigma_{1}^{3}$ satisfies the relation $\sigma_{1}^{3} a \leqslant a$. Note that if $A \in \mathbf{R}$, then the conditions S1, S3, S4 of Theorem 2.1 are satisfied with $\beta$ replacing $\nabla$. Moreover, if the relation $\alpha a \leqslant a$ is satisfied for each $a \in A$, then it follows from Proposition 3.2(ii) that equations S2 and ES3 hold in $A$ with $\beta$ replacing $\nabla$. Hence Proposition 2.3 yields that $A$ is a three-valued Lukasiewicz algebra. Thus we have:

3.4. Proposition. The class of three-valued Lukasiewicz algebras coincides with the equational subclass of $\mathbf{R}$ characterized by the equation (ER3): $a \vee \alpha a=a$.

The following results will play an important role in what follows:

3.5. Proposition. Let $A \in \mathbf{R}$ and $\mathbf{X}_{1}=\mathbf{X}_{1}(A)$ be the set of prime filters $P$ of $A$ such that $g(P) \subseteq P$. Then the maximal elements of $\mathbf{X}_{1}$ are exactly the filters of the form $\alpha^{-1}(F)$, where $F$ is an ultrafilter of the Boolean algebra $B(A)$.

Proof. Note first that the equality $\alpha \alpha a=\alpha a$ and Lemma 3.1 (i) imply that $\alpha^{-1}(F) \in \mathbf{X}_{1}$. Suppose now that $P \in \mathbf{X}_{1}$ and $\alpha^{-1}(F) \subseteq P$. Since $F=\alpha^{-1}(F) \cap$ $B(A)=P \cap B(A)$, it follows from Lemma 3.1 (i) that $P \subseteq \alpha^{-1}(F)$. Therefore, $\alpha^{-1}(F)$ is a maximal element in $\mathbf{X}_{1}$. To complete the proof, suppose that $P$ is a 
maximal element in $\mathrm{X}_{1}$, and set $F=P \cap B(A)$. Lemma 3.1 (i) yields $P \subseteq \alpha^{-1}(P)$ $=\alpha^{-1}(F)$, and since $P$ is maximal, we must have $\alpha^{-1}(F)=P$.

We shall denote by $\mathbf{G}_{R}$ the class of pairs $(X, g) \in \mathbf{G}_{K}$ satisfying the conditions: (rl) $\Lambda(U)=(U \cap g(U)] \cap[U \cap g(U))$ is open and increasing for each $U \in$ $\mathbf{D}(X)$, and (r2) for each $m \in \operatorname{Max} X_{1}$ and each $U \in \mathbf{D}(X)$, if $m \leqslant x$ for some $x \in g(U) \cap X_{2}$, then $m \in U$. If $(X, g)$ and $\left(X^{\prime}, g^{\prime}\right)$ are in $\mathbf{G}_{R}$, we shall say that $f$ : $X \rightarrow X^{\prime}$ is an R-function if it is a $\mathbf{G}$-function and $f\left(\operatorname{Max} X_{1}\right) \subseteq \operatorname{Max} X_{1}^{\prime}$. The category whose objects are the pairs in $\mathbf{G}_{R}$ and whose morphisms are the R-functions will be denoted by $\mathcal{G}_{R}$.

3.6. Lemma. Let $(X, g) \in \mathbf{G}_{R}$. Then the following properties hold for each $U \in \mathbf{D}(X)$ and each $m \in \operatorname{Max} X_{1}$ :

(i) $\Lambda(U) \in K(\mathscr{D}(X, g))$

(ii) If $x \in X$ is comparable with $m$, then $x \in \Lambda(U)$ if and only if $m \in U$. Moreover, we have that:

(iii) For each $x$ in $X$ there is exactly one $m_{x} \in \operatorname{Max} X_{1}$ that is comparable with $x$. Indeed, if $x \in X_{1}$, then $x \leqslant m_{x} \leqslant g\left(m_{x}\right) \leqslant g(x)$, and if $x \in X_{2}$, then $g(x) \leqslant m_{x}$ $\leqslant g\left(m_{x}\right) \leqslant x$.

(iv) If $\left(X^{\prime}, g^{\prime}\right)$ is also in $\mathbf{G}_{R}$ and $f: X \rightarrow X^{\prime}$ is an $\mathbf{R}$-function, then $f\left(m_{x}\right)=m_{f(x)}$ for each $x \in X$.

Proof. (i) Since $X$ is totally order-disconnected and $U \cap g(U)$ is closed in $X$, a compactness argument (see Priestley (1974, Proposition 1)) shows that $(U \cap g(U)]$ is closed, and it follows from the relations $g((U \cap g(U)])=[U \cap g(U))$ and $g([U \cap g(U)))=(U \cap g(U)]$, that $\Lambda(U)$ is an involutive closed set in $X$. Therefore $(\mathrm{r} l)$ implies that $\Lambda(U)$ is an involutive clopen increasing set in $X$, that is, that $\Lambda(U) \in K(\mathscr{D}(X, g))$.

(ii) Since (i) implies that $\Lambda(U)$ is simultaneously increasing and decreasing, we have that $x \in \Lambda(U) \Leftrightarrow m \in \Lambda(U)$. Thus to prove (ii) we need to show that $m \in U \Leftrightarrow m \in \Lambda(U)$. Suppose first that $m \in U$. Since $m \leqslant g(m)$, we have that $m \in U \cap g(U) \subseteq \Lambda(U)$. Suppose now that $m \notin U$. Then the maximality of $m$ in $X_{1}$ and condition ( $\left.\mathrm{r} 2\right)$ imply that $m \notin\left(U \cap X_{1}\right] \cup\left(g(U) \cap X_{2}\right]$, and since $\Lambda(U)$ $\subseteq\left(U \cap X_{1}\right] \cup\left(g(U) \cap X_{2}\right] \cup U$ we have that $m \notin \Lambda(U)$.

(iii) Suppose $x \in X_{1}$. Since $X_{1}$ is closed in $X$ (Cornish and Fowler (1979), Lemma 2.1), there is an $m \in \operatorname{Max} X_{1}$ such that $x \leqslant m$ (Priestley (1974), Proof of Proposition 3). Then we have that $x \leqslant m \leqslant g(m) \leqslant g(x)$. If $x \in X_{2}$, the above arguments applied to $g(x)$ yield $g(x) \leqslant m \leqslant g(m) \leqslant x$. Moreover, it follows from condition ( $\mathrm{r} 2)$ that each $x$ in $X$ can be comparable with at most one element of $\operatorname{Max} X_{1}$. 
(iv) By the definition of $\mathbf{R}$-functions it follows that $f(x)$ is comparable with $f\left(m_{x}\right) \in \operatorname{Max} X_{1}^{\prime}$.

Let $(X, g) \in \mathbf{G}_{R}$ and $U, V \in \mathbf{D}(X)$. It follows at once from (i) of the above lemma that for each $W \in K(\mathscr{D}(X, g)), U \subseteq \sim U \cup W=(X \backslash g(U)) \cup W$ if and only if $\Lambda(U) \subseteq W$. Consequently $\Lambda(U)$ is the least element in $H_{U}$, and since $\mathbf{G}_{R} \subseteq \mathbf{G}_{K}$, Proposition 3.2 yields that $\Lambda(U \cup V)=\Lambda(U) \cup \Lambda(V)$. Moreover, it follows from (ii) and (iii) of the above lemma that for each $x \in X, x \in \Lambda(U \cap V)$ $\Leftrightarrow m_{x} \in U \cap V \Leftrightarrow x \in \Lambda(U) \cap \Lambda(V)$. Therefore we also have that $\Lambda(U \cap V)=$ $\Lambda(U) \cap \Lambda(V)$, and the system $\mathscr{D}_{R}(X, g)=\langle\mathbf{D}(X), \cup, \cap, \sim, \Lambda, \varnothing, X\rangle \in \mathbf{R}$. Suppose now that $\left(X^{\prime}, g^{\prime}\right) \in \mathbf{G}_{R}$ and $f: X \rightarrow X^{\prime}$ is an $\mathbf{R}$-function. Define $\mathscr{D}_{R}(f)$ $=\mathscr{D}(f): \mathscr{D}\left(X^{\prime}, g^{\prime}\right) \rightarrow \mathscr{D}(X, g)$. By Lemma 3.6 we have that for each $U \in D\left(X^{\prime}\right)$, $x \in f^{-1}(\Lambda(U)) \Leftrightarrow U \ni m_{f(x)}=f\left(m_{x}\right) \Leftrightarrow m_{x} \in f^{-1}(U) \Leftrightarrow x \in \Lambda\left(f^{-1}(U)\right)$. Therefore $\mathscr{Q}_{R}(f)$ is an $\alpha$-De Morgan algebra homomorphism, and we have defined a functor $\mathscr{D}_{R}$ from the category $\mathcal{G}_{R}$ into the category $\mathcal{R}^{\text {op }}$.

Let $A \in \mathbf{R}$ and $(X, g)=\mathscr{P} r(A)$. Note first that Proposition 3.2 implies that $(X, g) \in \mathbf{G}_{K}$. Moreover, it follows from Lemma 3.1 that for each prime filter $P$ of $A$ we have that (1) $\alpha^{-1}(P) \subseteq g\left(\alpha^{-1}(P)\right)$ and (2) $P \subseteq \alpha^{-1}(P)$ or $\alpha^{-1}(P) \subseteq$ $\beta^{-1}(P) \subseteq P$. Since $\sigma(a)$ is increasing for each $a \in A$, (1) yields that $P \in \sigma(\alpha a) \Leftrightarrow$ $a \in \alpha^{-1}(P) \Leftrightarrow \alpha^{-1}(P) \in \sigma(a) \Leftrightarrow \alpha^{-1}(P) \in \sigma(a) \cap g(\sigma(a))$. Hence it follows from (2) that if $P \in \sigma(\alpha a)$, then $P \in \Lambda(\sigma(a))$. On the other hand the definition of $\alpha a$ implies that $\sigma(a) \cap g(\sigma(a)) \subseteq \sigma(\alpha a)$ and since $\sigma(\alpha a) \in K(\mathscr{D}(X, g))$, it follows that $\Lambda(\sigma(a)) \subseteq \sigma(\alpha a)$. Thus we have that $\sigma(\alpha a)=\Lambda(\sigma(a))$ for each $a \in A$, and, consequently, that $(X, g)$ satisfies condition $(\mathrm{r} 1) .(X, g)$ also satisfies (r2). Indeed, for each ultrafilter $F$ of $B(A)$ we have that $\alpha^{-1}(F) \in \sigma(a) \Leftrightarrow \alpha a \in$ $F \Leftrightarrow \beta \sim a=\sim \alpha a \notin F$. Moreover, it follows from Lemma 3.1(ii) that for each prime filter $P$ of $A, P \in g(\sigma(a)) \cap X_{2}$ if and only if $\sim a \notin P$ and $\beta^{-1}(P) \subseteq P$. Therefore $\alpha^{-1}(F) \subseteq P \in g(\sigma(a)) \cap X_{2}$ implies $\alpha^{-1}(F) \in \sigma(a)$, and applying Proposition 3.5 we obtain that $(X, g)$ satisfies (r2). Thus we have shown that if $A \in \mathbf{R}$, then $\operatorname{Pr}(A) \in \mathbf{G}_{R}$. Moreover, if $A^{\prime} \in \mathbf{R}$ and $h: A \rightarrow A^{\prime}$ is an homomorphism, we know that $\mathscr{P} r(h): X^{\prime} \rightarrow X$ is a G-function, and since for each ultrafilter $F$ of $B\left(A^{\prime}\right)$ we have that $(\mathcal{P} r h)\left(\alpha^{-1}(F)\right)=h^{-1}\left(\alpha^{-1}(F)\right)=(\alpha h)^{-1}(F)=$ $(h \alpha)^{-1}(F)=\alpha^{-1}\left(h^{-1}(F)\right)=\alpha^{-1}\left(h^{-1}(F) \cap B(A)\right)$, and since $h^{-1}(F) \cap B(A)$ is an ultrafilter of $B(A)$, it follows from Proposition 3.5 that $f$ satisfies the conditions of the definition of an $\mathbf{R}$-function.

Now it is easy to complete the proof of the following theorem, where $\mathscr{P} r_{R}$ denotes the restriction of the functor $\mathscr{P} r$ to the subcategory $\mathcal{R}$ of $\mathscr{Q}$ :

3.7. TheOREM. The categories $\mathcal{G}_{R}$ and $\Re^{\mathrm{op}}$ are naturally equivalent. More precisely, the composite functors $\mathscr{P} r_{R} \mathscr{D}_{R}$ and $\mathscr{D}_{R} \mathscr{P} r_{R}$ are naturally equivalent to the 
identity functors of $\mathcal{G}_{R}$ and $\mathscr{R}$ respectively. The corresponding unit is $\varepsilon$ and the co-unit $\sigma$.

Let $(X, g) \in \mathbf{G}_{R}$. We shall say that an involutive closed set $Y \subseteq X$ is an $\mathbf{R}$-set if $y \in Y$ implies $m_{y} \in Y$. Note that it follows from Lemma 3.6(iii) that an involutive closed set is an R-set if and only $[Y) \cap \operatorname{Max} X_{1} \subseteq Y$.

3.8. THEOREM. Let $A \in \mathbf{R}$ and $(X, g)=\operatorname{PP} r(A)$. Then the correspondence $Y \mapsto$ $\Theta(Y)$ establishes an isomorphism from the lattice of $\mathbf{R}$-sets of $X$ onto the order dual of the lattice of congruences of $A$.

Proof. Let $Y$ be an R-set of $X$. It follows from Lemma 3.6 that for each $U \in \mathbf{D}(X), x \in \Lambda(U) \cap Y$ implies $m_{x} \in U \cap Y$, and from this property and Lemma 1.1(i) it is easy to deduce that $\Theta(Y)$ is a congruence on $\mathscr{D}_{R}(X, \mathrm{~g})$ and that $Y=\varepsilon^{-1}\left(\mathscr{P} r(\pi)\left(\mathscr{P} r\left(\mathscr{D}_{R}(X, g) / \Theta(Y)\right)\right)\right)$.

On the other hand, let $\Theta$ be a congruence on $A$ and $\pi: A \rightarrow A / \Theta$ be the natural projection; since it is an immediate consequence of Lemma 3.6 that R-functions transform R-sets into R-sets, it follows from Lemma 1.1(ii) that $Y=$ $\operatorname{Pr}(\pi)(\mathscr{P r}(A / \Theta))$ is an R-set of $X$ and that $(a, b) \in \Theta$ if and only if $(\sigma(a), \sigma(b))$ $\in \Theta(Y)$.

Let $(X, g) \in \mathbf{G}_{R}$. For each $x \in X_{1}$ define $Y_{x}=\left\{x, m_{x}, g\left(m_{x}\right), g(x)\right\}$. It is plain that $Y_{x}$ is an R-set. We have the following possible cases:

(1) $x=m_{x}=g\left(m_{x}\right)=g(x)$,

(2) $x=m_{x} \leqslant g\left(m_{x}\right)=g(x)$,

(3) $x<m_{x}=g\left(m_{x}\right)<g(x)$,

(4) $x<m_{x}<g\left(m_{x}\right)<g(x)$.

The proofs of the following results are similar to those of Lemmas 2.6, 2.7 and Theorem 2.8 respectively:

3.9. Proposition. Let $A \in \mathbf{R}$ and $(X, g)=\mathscr{P} r_{R}(A)$. Then:

(i) $A$ is a subdirect product of the family of algebras

$$
\left\{\mathscr{9}_{R}\left(Y_{x}, g_{x}\right)\right\}_{x \in X_{1}}
$$

(ii) If $\left|Y_{x}\right|=j$, then $\mathscr{D}_{R}\left(Y_{x}, g_{x}\right) \cong L_{j+1}, 1 \leqslant j \leqslant 4$.

3.10. THEOREM. The subdirectly irreducible algebras in the equational class $\mathbf{R}$ are $L_{i}$ for $2 \leqslant i \leqslant 5$. The simple algebras are $L_{2}$ and $L_{3}$.

Denote by $\mathbf{R}_{i}$ the equational subclass of $\mathbf{R}$ generated by $L_{i}$, for $2 \leqslant i \leqslant 5$. It follows from Theorems 3.8, 3.10 and 1.1 that the lattice of equational subclasses 
of $\mathbf{R}$ is the finite distributive lattice having as join-irreducible elements the classes $\mathbf{R}_{i}$, ordered as follows: $\mathbf{R}_{2} \leqslant \mathbf{R}_{3}, \mathbf{R}_{4} \leqslant \mathbf{R}_{5}=\mathbf{R}$, and $\mathbf{R}_{3}$ not comparable with $\mathbf{R}_{4}$. Thus the lattice of equational subclasses of $\mathbf{R}$ is isomorphic to the lattice obtained by adding a new zero and a new unit to the Boolean algebra with two atoms.

The proof of the next theorem is analogous to that of Theorem 2.9, taking into account Proposition 3.4:

3.11. THEOREM. The class of three-valued Lukasiewicz algebras coincides with $\mathbf{R}_{3}$. Thus, the class of three-valued Lukasiewicz algebras can be characterized as the class of semi-simple regular a-De Morgan algebras. Moreover, it coincides with the intersection $\mathbf{R} \cap \mathbf{S}$.

\section{Lukasiewicz algebras}

We shall consider now algebras that are simultaneously regular $\alpha$-De Morgan algebras and involutive Stone algebras, that is, algebras $\langle A, \vee, \wedge, \sim$, $\alpha, \nabla, 0,1\rangle$ of type $(2,2,1,1,1,0,0)$ such that $\langle A, \vee, \wedge, \sim, \alpha, 0,1\rangle \in \mathbf{R}$ and $\langle A, \vee, \wedge, \sim, \nabla, 0,1\rangle \in \mathbf{S}$.

The equational class of such algebras will be denoted by $\mathbf{Q}$ and the corresponding category by 2 . Moreover, $\mathbf{L}_{n}(n \geqslant 2)$ will denote the (equational) class of $n$-valued Lukasiewicz algebras and $\mathscr{E}_{n}$ the corresponding category.

Note that the algebras $L_{n} \in \mathbf{Q}$ for each $n \geqslant 2$, and that if $A \in \mathbf{L}_{n}$, then the reduct $\left\langle A, \vee, \wedge, \sim, \sigma_{t}{ }^{n}, \sigma_{n-1}^{n}, 0,1\right\rangle \in \mathbf{Q}$, where $t=(n-1) / 2$ if $n$ is odd and $t=n / 2$ if $n$ is even. In particular, for $n=5$, we have that $\langle A, \vee, \wedge, \sim$, $\left.\sigma_{2}^{5}, \sigma_{4}^{5}, 0,1\right\rangle \in \mathbf{Q}$ and that the operators $\sigma_{2}^{5}$ and $\sigma_{4}^{5}$ satisfy the relation $a \wedge \sigma_{4}^{5} \sim a$ $\leqslant b \vee \sim \sigma_{2}^{5} b$, for each $a, b$ in $A$. Note that this relation corresponds to the case $i=1$ in axiom L14 of the definition of $n$-valued Lukasiewicz algebras given in Cignoli (1970) (or axiom L10 in the definition given in Balbes and Dwinger (1974, page 219), where $D_{i}=\sigma_{n-i}$ ). Conversely, if $A \in \mathbf{Q}$ and the relation $a \wedge \nabla \sim a \leqslant b \vee \sim \alpha b$ holds in $A$, then $\langle A, \vee, \wedge, \sim, \triangle, \alpha, \beta, \nabla, 0,1\rangle \in \mathbf{L}_{5}$. Indeed, if we set $\sigma_{1}^{5}=\triangle, \sigma_{2}^{5}=\alpha, \sigma_{3}^{5}=\beta$ and $\sigma_{4}^{5}=\nabla$, it is plain that they satisfy the axioms L1-L5 and L10 from the characterization of Lukasiewicz algebras given in Cignoli (1970) (or axioms L1-L9 in Balbes and Dwinger (1974, page 219), where $D_{i}=\sigma_{5-i}$ ). Moreover, the inequality $a \wedge \sim \sigma_{2}^{5} a \wedge \sigma_{3}^{5} b \leqslant b$ is equivalent to $a \wedge \sim \alpha a \leqslant b \vee \alpha \sim b$ (see (ii) of Proposition 3.2), and each of the inequalities $a \wedge \sim \sigma_{1}^{5} a \wedge \sigma_{2}^{5} b \leqslant b$ and $a \wedge \sim \sigma_{3}^{5} \wedge \sigma_{4} b \leqslant b$ is equivalent to $a \wedge \nabla \sim a \leqslant b \vee$ $\sim \alpha b$. Thus, axiom L14 from Cignoli (1970) (or axiom L10 of Balbes and Dwinger (1974)) is also satisfied. 
Since it was shown in Cignoli and de Gallego (1981) that the class of four-valued Lukasiewicz algebras can be identified with the equational subclass of $\mathbf{L}_{5}$ characterized by the equation $\alpha a=\beta a$, and the class of three-valued Lukasiewicz algebras with the equational subclass of $\mathbf{L}_{5}$ characterized by the equation $\alpha a=\sim \nabla \sim a$, we have the following:

4.1. Propositron. The class of five-valued Lukasiewicz algebras coincides with the equational subclass of $\mathbf{Q}$ characterized by the equation (EQ5) $(a \wedge \nabla \sim a) \vee(b$ $\vee \sim \alpha b)=b \vee \sim \alpha b$. The class of four-valued Lukasiewicz algebras coincides with the equational subclass of $\mathbf{Q}$ characterized by the equations (EQ5) and (EQ6) $\alpha a=\beta a$. The class of three-valued Lukasiewicz algebras coincides with the equational subclass of $\mathbf{Q}$ characterized by the equation (EQ3) $\alpha a=\sim \nabla \sim a$.

Let $\mathbf{G}_{Q}=\mathbf{G}_{S} \cap \mathbf{G}_{R}$ and $\mathcal{G}_{Q}$ be the category whose objects are the elements of $\mathbf{G}_{Q}$ and whose morphisms are the functions that are simultaneously morphisms in $\mathcal{G}_{R}$ and $\mathcal{G}_{S}$.

If $(X, g) \in \mathbf{G}_{Q}$, define $\mathscr{D}_{Q}(X, g)=\langle\mathbf{D}(X), \cup, \cap, \sim, \Lambda, \nabla, X, \varnothing\rangle$ and if $f$ is a morphism in $\mathcal{G}_{Q}$, set $\mathscr{D}_{Q}(f)=\mathscr{D}(f)$. Then, if $\mathcal{P r}_{Q}$ denotes the restriction of the functor $\mathcal{P} r$ to the subcategory $\mathcal{Q}$ of $\mathcal{Q}$, it follows from Theorems 2.3 and 3.7 that:

4.2. THEOREM. The categories $\mathcal{G}_{Q}^{o p}$ and $\mathcal{Q}$ are naturally equivalent. More precisely, the composite functors $\mathscr{P} r_{Q} \mathscr{D}_{Q}$ and $\mathscr{D} Q \mathcal{P} r_{Q}$ are naturally equivalent to the respective identity functors. The corresponding unit is $\varepsilon$ and the co-unit $\sigma$.

If $(X, g) \in \mathbf{G}_{Q}$, we shall say that $Y \subseteq X$ is a Q-set if it is both an $\mathbf{R}$-set and an S-set. It follows at once from Theorems 2.5 and 3.8 that if $A \in \mathbf{Q}$, then the correspondence $Y \mapsto \Theta(Y)$ establishes an isomorphism from the lattice of $\mathbf{S}$-sets in $\operatorname{Pr}(A)$ onto the order dual of the lattice of congruences on $A$. If for each $x \in X_{1}$ we set $Y_{x}=\left\{x, m_{x}, n_{x}, g\left(m_{x}\right), g\left(n_{x}\right), g(x)\right\}$ and $g_{x}$ is the restriction of $g$ to $Y_{x}$, then the arguments used in the proofs of Lemmas 2.6 and 3.9 show that $\left(Y_{x}, g_{x}\right) \in \mathbf{G}_{Q}$ and that $\mathscr{D}_{Q}(X, g)$ is a subdirect product of the family $\left\{\mathscr{l O}_{Q}\left(X_{x}, g_{x}\right)\right\}_{x \in X_{1}}$.

By examining the possible cases, we get that $\left|Y_{x}\right| \leqslant 6$ and that if $\left|Y_{x}\right|=j$, then ${ }^{2} Q_{Q}\left(Y_{x}, g_{x}\right) \cong L_{j+1}$, for $1 \leqslant j \leqslant 6$. Thus we can establish:

4.3. THEOREM. The subdirectly irreducible algebras in the equational class $\mathbf{Q}$ are $L_{i}$ for $2 \leqslant i \leqslant 7$. The simple algebras are $L_{i}$ for $2 \leqslant i \leqslant 5$.

Let $\mathbf{Q}_{i}$ denote the equational subclass of $\mathbf{Q}$ generated by the algebra $L_{i}$, $2 \leqslant i \leqslant 7$. It follows from the above results and Theorem 1.1 that the lattice of 
equational subclasses of $\mathbf{Q}$ is given by the following Hasse diagram:

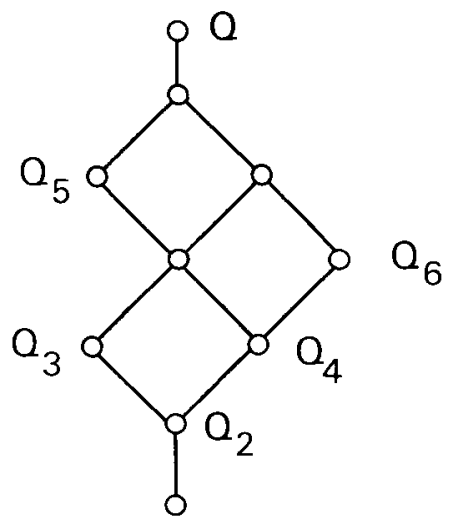

Now it is easy to prove the following:

4.4. THEOREM. The class $\mathbf{L}_{i}$ coincides with the class $\mathbf{Q}_{i}$ for $i=2,3,4$ and 5. In particular, the class of five-valued Lukasiewicz algebras can be characterized as the class of semisimple algebras in $\mathbf{Q}$.

4.5. CoRollary. The proper, non-trivial equational subclasses of $\mathbf{L}_{5}$ are $\mathbf{L}_{2}, \mathbf{L}_{3}$, $\mathbf{L}_{4}$ and the class $\mathbf{H}$ generated by $L_{3}$ and $L_{4}$. The class $\mathbf{H}$ is characterized by the equation $(a \wedge \sim a) \vee\left(b \vee \sim \sigma_{4}^{5} b\right)=b \vee \sim \sigma_{4}^{5} b$.

\section{References}

R. Balbes and P. Dwinger (1974), Distributive lattices (University of Missouri Press, Columbia, Missouri).

A. Bialynicki-Birula and H. Rasiowa (1957), 'On the representation of quasi-Boolean algebras', Bull. Acad. Polon. Sci. Cl. III 5, 259-261.

R. Cignoli (1970), Moisil algebras (Notas de Lógica Matemática No. 27, Universidad Nacional del Sur, Bahia Blanca).

R. Cignoli (1974), Topological representation of Lukasiewicz and Post algebras (Notas de Lógica Matemática No. 33, Universidad Nacional del Sur, Bahía Blanca).

R. Cignoli (1979), 'Coproducts in the categories of Kleene and Three-valued Lukasiewicz algebras', Studia Logica 38, 237-245.

R. Cignoli and M. S. de Gallego (1981), 'The lattice structure of some Lukasiewicz algebras', Algebra Universalis 13, 315-328.

W. H. Cornish (1975), 'On H. Priestley's dual of the category of bounded distributive lattices', Mat. Vestnik 12(27), 329-332.

W. H. Cornish and P. R. Fowler (1977), 'Coproducts of De Morgan algebras', Bull. Austral. Math. Soc. 16, 1-13. 
W. H. Cornish and P. R. Fowler (1979), 'Coproducts of Kleene algebras', J. Austral. Math. Soc. Ser A 27, 209-220.

B. A. Davey (1978), 'Subdirectly irreducible distributive double $p$-algebras', Algebra Universalis 8 , 73-88.

B. A. Davey (1979), 'On the lattice of subvarieties', Houston J. Math. 5, 183-192.

B. Jónsson (1967), 'Algebras whose congruence lattices are distributive', Math. Scand. 21, 110-121.

T. Katriňak (1974), 'Injective double Stone algebras', Algebra Universalis 4, 259-267.

H. A. Priestley (1970), 'Representation of distributive lattices by means of ordered Stone spaces', Bull. London Math. Soc. 2, 186-190.

H. A. Priestley (1972), 'Ordered topological spaces and the representation of distributive lattices', Proc. London Math. Soc. (3) 24, 507-530.

H. A. Priestley (1974), 'Stone lattices: a topological approach', Fund. Math. 84, 127-143.

H. A. Priestley (1975), 'The construction of spaces dual to pseudocomplemented distributive lattices', Quart. J. Math. Oxford Ser. 26, 215-228.

J. Varlet (1968), 'Algèbres de Lukasiewicz trivalentes', Bull. Soc. Roy. Sci. Liège 36, 399-408.

Instituto de Matemática

Universidade Estadual de Campinas

13.100-Campinas-São Paulo-Brazil 